\title{
BIBLICAL INDEX
}

\section{Old Testament}

Genesis

$\begin{array}{ll}4 & 121 \\ 4: 10 & 155 \\ 9: 12-15 & 180-1 \\ 10: 22-4 & 121 \\ 28: 12 & 162-3 \\ 36: 2 & 121\end{array}$

Exodus

$$
\begin{array}{ll}
15: 27-16: 10 & 24 \\
20: 6 & 79-80 \\
28: 36-40 & 61
\end{array}
$$

Numbers

$$
\begin{array}{ll}
5: 23-8 & 94 n .5 \\
6: 24-6 & 60
\end{array}
$$

Deuteronomy

$$
6: 8-9 \quad 60
$$

Judges

$\begin{array}{ll}1: 15 & 181 \\ 11: 33 & 121\end{array}$

1 Samuel

$6: 18$

1 Kings

\begin{tabular}{rl}
\multicolumn{1}{c}{$\begin{array}{l}\text { 2 Kings } \\
15: 29\end{array}$} & 155 \\
$20: 13$ & 121 \\
& $122-3$
\end{tabular}

3 Esdras

4 Esdras

Tobit

Judith

$$
1: 1
$$

Psalms
121

$$
130,164
$$$$
130,164
$$

164

164

121

48-9, 131-8, 163, plates 5,7

superscriptions $134-8$

$$
135^{-6}
$$

$\begin{array}{ll}11: 2 & 183 \\ 38 & 132 \\ 65: 2 & 33 \\ 69: 2 & 39 \\ 97 & 132 \\ 109 & 132 \\ 117 & 22 \\ 117: 25^{-6} & 47 \\ 118: 130 & 165 \\ 132 & 186 \\ \text { dom Books } & 163 \\ \text { verbs } & \end{array}$

Proverbs

$\begin{array}{ll}14: 13 & 27 \\ 23: 35 & 183\end{array}$

Ecclesiastes

9:14 $\quad 183$

Song of Songs 122,131

1:1

168-170, figure 8

5:7

183

Wisdom $\quad 164$

Ecclesiasticus $\quad 164$

Isaiah

6:3 71

39:2 $\quad 123$

61:1 61, 152

63:1 29-30, 178

Jeremiah $\quad 164$

Lamentations 130-1

Daniel

2:37
Zechariah

9:9

1 Maccabees

29, 176-8, 202

2 Maccabees $\quad 164$

7

186 


\section{New Testament}

Gospels $\quad 163$

Matthew

$\begin{array}{ll}\text { 1:1-21 } & 106 \mathrm{n} .113 \\ 2: 1-12 & 89 \\ 3: 2 & 164 \\ 3: 13-17 & 106 \mathrm{n} .113 \\ 4: 17 & 164 \\ 5: 36 & 79 \\ 6: 3 & 180 \\ 20: 17-19 & 89,106 \mathrm{n} .117 \\ 21: 1-9 & \text { chapters } 1,4 \\ 21: 1 & 166-70 \\ 21: 1-2 & 172-5 \\ 21: 5 & 28-9,175^{-8} \\ 21: 8 & 179-80 \\ 21: 9 & 47 \\ 21: 15-16 & 21,34 \\ 23: 5 & 94 \text { n.4 } \\ 23: 35 & 121 \\ 26: 1-27: 61 & 44,106 \text { n.113 } \\ 27: 11-26 & 34 \\ & \end{array}$

Mark

$8: 15^{-26} \quad 89$

11:1-11 14, 166-7

11:28 $\quad 182$

14:1-15:46 106n.113

16:1-7 106n.113

16:14-20 $\quad 89,106 \mathrm{nn} .113 \& 11$

$16: 15 \quad 152,160,172$

Luke

$\begin{array}{ll}1: 5-17 & 106 \mathrm{n} .113 \\ 1: 26-38 & 89,106 \mathrm{nn} \\ 1: 35 & 184-5 \\ 2: 10 & 161 \\ 3: 21-4: 1 & 106 \mathrm{n} .113 \\ 3: 22 & 61\end{array}$

$\begin{array}{ll}4: 16-30 & 152 \\ 8: 11 & 165^{-6} \\ 11: 28 & 160-1 \\ 12 & 186 \\ 10: 1 & 64 \\ 19: 1-10 & 35 \\ 19: 28-38 & 14 \\ 19: 37 & 26,33 \\ 19: 38 & 33 \\ 22: 1-23: 53 & 106 n .113 \\ & 106 \text { n.113 } \\ 1: 1-14 & 89,92,106 \text { n.113, } \\ & 203 \\ 1: 29 & 31 \\ 8: 12 & 30 \\ 8: 47 & 160 \\ 10: 16 & 177 \\ 11: 47-53 & 31,37-8 \\ 12: 12-16 & 14,24 \\ 12: 13 & 19\end{array}$

Acts of Apostles

4:11 60

Pauline Epistles 163

Romans

$\begin{array}{ll}1: 16 & 65 \\ 12: 9 & 173\end{array}$

Colossians

3:12 $\quad 182$

Laodiceans

(apocryphal) 164

James

\begin{tabular}{cc}
\multicolumn{1}{c}{$5: 12$} & 79 \\
Jude & 164 \\
Revelation & \\
$4: 8$ & 71 \\
$12: 4$ & 185 \\
$16: 15$ & 182
\end{tabular}




\section{Cambridge}

Cambridge University Library

Dd.1.14 Appendix

Dd.5.52 Appendix

Dd.8.12 116, 117-18, 143n.17, 144n.18, Appendix

Dd.10.29 115-16, Appendix

Dd.12.47 Appendix

Dd.13.6 131, Appendix

Ee.1.9 Appendix

Ee.1.16 143n.17, Appendix

Ee.2.23 113, 129, 131, Appendix

Ee.6.26 144n.28, Appendix

Hh.1.3 143n.13, Appendix

Ii.6.22 131

Kk.2.6 57n.82, 200

Mm.1.2 144n.18, Appendix

Mm.3.2 Appendix

Corpus Christi College

286 (St Augustine Gospels) 93

Emmanuel College

$$
252
$$

21-22

St. John's

A1 128

B14 77-8

C24 138, Appendix

I28 138, 144n.28, Appendix

N1 143n.13, Appendix

N8 131, 144n.28, Appendix

N11 Appendix

Trinity College

$$
\text { O.1.71 77-8 }
$$

\section{Canterbury}

Canterbury Cathedral

$$
\text { Law Society } 3 \quad 143 n .13,144^{-5} \text { n.28 }
$$


Edinburgh

Edinburgh University Library
2
Appendix
4
124-6 24-5, plate 5, Appendix

\section{Glasgow}

Glasgow University Library

Gen 999 53n.23

\section{London}

The British Library

Additional 5,160 Appendix

Additional 11,842 113, Appendix

Additional 15,253 126, 138, 143n.17, Appendix

Additional 15,452 Appendix

Additional 22,573 87-8

Additional 35,085 113-14, 145n.28, Appendix

Additional 39,629 Appendix

Additional 49,598 21

Additional 57,534 53n.30,56n.79

Arundel 78

Appendix

Arundel 83

173-5, figure 9

Arundel 303

113-14, 133, Appendix

Arundel 311

Appendix

Burney 1

Appendix

Burney 2

143n.17, Appendix

Burney 5

131, Appendix

Burney 6

Appendix

Burney 7

Appendix

Burney 9

Appendix

Burney 10

Appendix

Burney 11

133, Appendix

Cotton Galba E.iv 70-1

Egerton $656 \quad 77$

Egerton $2867 \quad$ Appendix

Harley 613 Appendix

Harley 1661 Appendix

Harley $2787 \quad 66$

Harley 2813 Appendix

Harley 2942 53n.30,56n.79

Harley $4919 \quad 66$

Lansdowne 438 Appendix 
Lansdowne 453 Appendix

Royal 1 A.xix $\quad 143-4$ n.17, Appendix

Royal 1 B.iii Appendix

Royal 1 B.viii 131, Appendix

Royal 1 B.x $\quad 117-18,144$ n.18

Royal 1 B.xi $\quad 74$

Royal 1 B.xii Appendix

Royal 1 D.i 133, Appendix

Royal 1 D.iii $\quad 74-5$

Royal 1 D.iv Appendix

Royal 1 E.i Appendix

Royal 1 E.ix $\quad$ 125-6, Appendix

Royal 2 F.iv $\quad 122$

Royal 9 A.vii $\quad 88-9$

Royal 9 A.xii $\quad 88-9$

Sloane $2478 \quad 37-41$

Stowe $1 \quad 144$ n.18, Appendix

Stowe $15 \quad 86-7$, plate 3

Guildhall Library

04645 89, 106-7n.120

4158A 67, 113

Lambeth Palace Library

533

144nn.18\&28, Appendix

$534 \quad$ 144nn.18\&28, Appendix

$756 \quad$ Appendix

$1362 \quad 67$

Register of Thomas Arundel, Archbishop of Canterbury, i

80

Victoria and Albert Museum

L. 2060-1948 115

Reid $21 \quad 126-7,130$, plate 6, Appendix

\section{New York}

Pierpont Morgan Library
M. 708
$73-4$, Plate 2
M. 709
$73-4$

\section{Oxford}

The Bodleian Library

Bodley $687 \quad$ chapter 4

Lat. bibl. e.7 132-3, 143n.13, 145n.28, 149n.66, plate 7

Lat. liturg. d. $42 \quad 25,35$, plate 1 
Laud Misc. 77 chapter 4

Laud Misc. $291 \quad 63-4$

New College Archives

3914

\section{Paris}

Bibliothèque de l'Arsénal

97

119-23

Bibliothèque nationale de France

Cod. Graec. $510 \quad 61$

\section{San Marino (CA)}

Huntington Library

HM 26061 $114,126,143$ n.13, 145n.28, plate 4

\section{York}

Minster Library and Archives

Additional 1 (York Gospels) 89-90 


\section{GENERAL INDEX}

Alain of Lille, Summa de arte praedicatoria $162-4$

Alexander of Ashby, De artificioso modo predicandi 163

Augustine of Hippo 161-3, 186, 209

Bacon, Roger 7-8

Ball, John, leader of 1381 Revolt 205

Barking Abbey (London) 20

Basevorn, Robert, Forma praedicandi $163-5,191$. 22

Bede $60,118,136$

Beleth, John, Summa de ecclesiasticis officiis 23,63

Bériou, Nicole 153

Bernard of Clairvaux 179

Bernardino of Siena 194n.59

Bible For specific episodes see biblical index

biblical glossaries

Jerome's Liber interpretationis 119-23

Interpretations of Hebrew Names 118-24, 130, 138, 140, 166-70, 188, 201

Isidore's 'Adam figuram Christi' 122

biblical mnemonics $8-9,19,112$, $115,130,133,138-40,187$

Cursus evangelii 89 early printed English

Bibles 93-4, 138-40

parish ownership vide parish as single-volume $66,112,120$ order of books 109-110 private ownership 68, 109, 207 shift from narrative to text 165 , $187-8,200-2$

Torah scrolls 84, 93

tripartite division in monasteries 70

Wycliffite Bibles 150n.74, 208

Black Death 105n.103

Books of Hours 4, 89, 92, 112, 132, 134,207

Brinton, Thomas 155

Caiphas, Speech of 31,37-41, 205

Canterbury, Christ Church 70-2

Chadd, David 18,66

Chaucer, Geoffrey, Canterbury

Tales 81-2, 90

Chester, Principality 106n.110

Chester, St Weburg abbey 85

Comestor, Peter 63-4, 77

Coverdale, Miles 115

Cumdach 60

Cursor mundi 83

d'Esneval, Amaury 118, 119, 122-3, $145 n \cdot 31$

Dahan, Gilbert 110, 118

Daniélou, Jean 8 de Hamel, Christopher 5, 132

De sex alis cherubim 116-17

Debray, Régis 3

Drax, Austin priory (Yorkshire) 85

Duffy, Eamon 3, 26

Durand, William, Rationale divinorum officiorum 


$$
\begin{aligned}
& 23-25,33,34,37,39-41, \\
& 61,64
\end{aligned}
$$

Earl of Toulouse (verse romance) 83

Edmund of Almain, second Earl of

Cornwall (d. 1300) 100n.56

Edward II 155

Egeria (pilgrim to Jerusalem) 19,

$$
\text { 21, } 41
$$

Elizabeth de Burgh, Lady of Clare 109

Eusebian canons 74, 110, 202

Farnylawe, Thomas, Chancellor of

$$
\text { York (d. 1379) 151n.87 }
$$

Genette, Gérard 5

Gesta Romanorum 46

Glastonbury Abbey (Somerset) 72-3

Gospel book (textus) 25-6, chapter 2 English textus 100n. 56

sine libro 71

survival 90, 207

tabula textus (bindings) 68, 71 with relics $72-3$

Gospel of Nicodemus 31, 46

Gratian, Decretum 79-80

Gregory the Great 161-3

Gutenberg Bible 138-9, figure 6

Harrowing of Hell vide Gospel of

Nicodemus

Havelok the Dane (verse romance) 82-3

Henry de Harkeley, Chancellor of the University of Oxford (d. 1317) 155

Henry III 75-6, 83

Henry V (royal entry after Agincourt) $35^{-6}$

Hereford 19, 20

Hide Abbey (Hants.) 101n.69

Hubert de Burgh $75^{-6}$
Hugh of St Cher 128

Hugh of St Victor 133

Humbert of Romans 179

Isabella of Castile, daughter of Pedro the Cruel 109

Jerome 6o, 110, 118-21, 130, 136, 142 n. $4,176,199$

Jerusalem 19, 21-4, 167, 170

Jews 34, 123, 155

Abraham son of Deulecresse the Jew of Norwich 84

community of York (and Meyrot, its sergeant) 84

Jewish Bible in Christian use 128 Jewish oath vide Oath

textus pawned at the Jewish community of Cambridge 75

John de Borw (d. 1384) 69, 99n.47

John de Grandisson, Bishop of Exeter 68

John Edward, Chaplain suspected of Lollardy 80

John of Bologna, Summa notarie $78-9$

John of Ufford, canon of Sarum and Lincoln (d. 1375) $151 n .87$

Judith of Flanders 74

Kempe, Margery 43

Kiss $65,97 n .28,78,168-9$

Lanfranc, Constitutions 19, 20, 25

Langland, William, The Vision of

Piers Plowman 46-7, 200

Langton, Stephen 6, 127

Late Medieval Bible chapter 3

(especially 109-12), 154-5, $164,166,168,180$, 200-2, 207-8, Appendix 
Lay Folk Mass Book 64, 97n.29

Light, Laura 110

Lincoln 19, 51n.8, 21, 22

Liturgy

and antiquity 36, 47-8, 203, 208

chants, prayers and hymns

for Palm Sunday see chapter 1

Ave Maria gratia plena

(responsory) $184-5$

Deus in adiutorium meum

(versicle) 38-9

exultet (Easter Proclamation) 74

Lesser doxology 137

sanctus (hymn) 71

Super choros angelorum

(versicle) 185

church councils 61

consecration of bishops 61,81

emotional experience $15,19,26$, $33,35^{-6}, 37,41-4,63^{-6}$

and law $62,78,79,82-3$

modern Psalmody 150n.71

musical qualities $27-8,31,40-1$, 56n.78, 46, 47, 133-4

Ordinary of the Mass $39,63-66$ and preaching 159-6o, 178,

$$
\text { 184-6, 199-201 }
$$

sensory experience $63^{-6}$

Locke, John 127-8, 198

Lollardy 43, 80, 92, 167, 206

Malcolm III of Scotland 101n.73

Malone, Carolyn 21, 23, 52n.19

Margaret of Scotland 101n.73

Martin of St Cross $68-9$

McGurk, Patrick 73-4

Monk Bretton (Yorkshire) 143-4n.17

Newton Longville, Priory (Buckinghamshire) 77

Nigel, Bishop of Ely (d. 1169) 73, 75
Oaths 6o, 74-94

Barak H. Obama on Abraham Lincoln's Bible 94

as breach of Third Commandment $79-80$

Burgh 76

inability of priests to swear 78 , 80

Jewish 83-4

Lollard 206

oath-books

lack of Bibles 85

liturgical books 82-3

preferred Gospel texts $88-9$

supply 84,85

tripartite understanding of ritual $77-8$

Odo of Cheriton 6, 156 passim

Palazzo, Eric 72

Palm Cross 20, 27, 32, 36

Palms 24-25, 36, 39, 49, 65

Paris Bible (vide also Late Medieval

Parish

Bible) 109-110

Book requirements 68

biblical iconography in 21-2, 44, figures 3,4

liturgy in $15^{-18,35,66}$

ownership of Bibles 66-9, 109, $141,151 n .87$

ownership of oath-books 86

Peasants' Revolt (1381 Revolt) 155, 205

Pecock, Reginald, The Repressor of Over Much Blaming of the Clergy 42-3

Peter des Roches $75^{-6}$

Peter the Deacon of Monte Cassino, De locis sanctis 24

Peterborough Abbey (now

Cathedral of ss Peter, Paul, 
and Andrew) 60

Pfaff, Richard W. 112-13, 143n.13

Philip of Harveng, Commentaria in

Cantica Canticorum 168

Preaching chapter 4

Aesthetics of division 175, 181-2, 188,199

and contemporary events 155

and exegesis 153, 161, 162, 164-5,

and Late Medieval Bible 200

macaronic or billingual 157

New Form 123, 153-5, 163,

$$
185^{-6}
$$

Psalters 60 (talismanic use), 67-8, $70-2$

Reading Abbey (and Alured of Dover, sacrist) 116-17

Remigius of Auxerre 116, 118-19

Richard Coeur de Lion (verse romance) $80-1$

Richard II 155

Ricoeur, Paul 48-9, 152

Rochester, Cathedral and Benedictine Priory of St Andrews 72, 74-5

Rouse, Richard H. and Mary A. 116, $118,127,145$ n.31

Rubin, Miri 3

Rupert of Deutz 96n.18, 64

Saenger, Paul 132-3

Sarum

Cathedral 70, 75
Liturgical use chapter 1, 66

(hegemony), 184

visitations 20,69

Simon of Sudbury, Archbishop of

Canterbury 155, 206

Smith, Wilfred Cantwell 202

Stock, Brian 3, 48, 69

Theodulf of Orléans 6, 33, 124

Thomas Becket (Psalter, cult, iconography) 71-2, 155

Thomas of Woodstock, son of Edward III 109

Thorton-on-Humber, Austin

Abbey (North

Lincolnshire) 115

Vezin, Jean 60-1, 72, 73

Waldeby, John 158 passim

Watking, Dom Aelerd 67-8

Wells Cathedral 21, 23,37

Wimbledon, Thomas 155

Wyclif,John 6, 8, 80, 92, 165-6, 205-6

York 19, 20, 65, 68

Augustinian Friary 158

ecclesiastical court 85

Jewish community vide Jews

Minster 89-90

St Mary's 25

St Nicholas $151 n .87$

Ywain and Gawain (English rendering) 83 